PROCEEDINGS OF THE

AMERICAN MATHEMATICAL SOCIETY

Volume 125, Number 2, February 1997, Pages 355-361

S 0002-9939(97)03724-6

\title{
KRULL DIMENSION OF MODULES OVER INVOLUTION RINGS. II
}

\author{
K. I. BEIDAR, E. R. PUCZYŁOWSKI, AND P. F. SMITH
}

(Communicated by Ken Goodearl)

\begin{abstract}
Let $R$ be a ring with involution and invertible 2 , and let $\bar{S}$ be the subring of $R$ generated by the symmetric elements in $R$. The following questions of Lanski are answered positively:

(i) Must $\bar{S}$ have Krull dimension when $R$ does?

(ii) Is every Artinian $R$-module Artinian as an $\bar{S}$-module?
\end{abstract}

Throughout this paper $R$ is a ring with involution $*$ in which 2 is invertible. We shall denote by $S(R)$ the set of symmetric elements of $R$ and by $\bar{S}(R)$ the subring of $R$ generated by $S(R)$.

All modules in this paper are assumed to be unital left modules. Given a ring $A$ and an $A$-module $M$, the Krull dimension of $M$ will be denoted by $k_{R}(M)$.

Chain conditions in rings with involution were studied in a number of papers (see, for example, [2], [4], [8], [9], [10], [11]). In [10] Lanski conjectured that if $R$ has Krull dimension, then $\bar{S}(R)$ also must have Krull dimension. He raised implicitly a more general question, namely whether every $R$-module with Krull dimension has the same Krull dimension as an $\bar{S}(R)$-module. He frankly admitted that he did not know the answer even in the case of Artinian modules. In [2] the general question was affirmatively answered in the case when $R$ is Noetherian with respect to two-sided $*$-ideals. The main aim of this paper is to prove the following:

A. Every Artinian $R$-module is Artinian as an $\bar{S}(R)$-module.

B. If $R$ is a ring with Krull dimension and $M$ is an $R$-module, then $k_{R}(M)=$ $k_{\bar{S}(R)}(M)$.

The latter settles in particular Lanski's conjecture above.

The idea of the proof is similar to that in [2]. However we now apply some additional deep results and change the technique of the proof in some crucial points.

Note that if elements of $S(R)$ commute, then $S(R)=\bar{S}(R)$. In [1] Amitsur proved that if $\bar{S}(R)$ is a PI ring (e.g. if it is commutative), then so is $R$.

For every $*$-ideal $I$ of $R, R / I$ is a ring with involution in a natural way and $S(R / I)=(S(R)+I) / I$. Indeed, if $r+I \in S(R / I)$, then $r^{*}+I=r+I$. Hence $r+I=\left(r+r^{*}\right) / 2+I \in S(R)+I$. The other inclusion is clear.

An $R$-module $M$ will be called $*$-faithful if for every non-zero $*$-ideal $I$ of $R$, $I M \neq 0$.

Received by the editors August 23, 1995.

1991 Mathematics Subject Classification. Primary 16W10, 16P60.

The research of the second author was partially supported by KBN grant 2 P301 03506 .

(C)1997 American Mathematical Society 
Note that if $M$ is an $R$-module and $T$ is the sum of all $*$-ideals of $R$ annihilating $M$, then $M$ is a $*$-faithful $(R / T)$-module. Obviously the Krull dimensions of $M$ over $R$ and over $R / T$ coincide. We shall need the following

Proposition 1. If $R$ is a PI ring having a $*$-faithful module $M$ with Krull dimension, then the prime radical $\beta(R)$ of $R$ is nilpotent.

Proof. In [12] Markov proved that if a PI ring $A$ has a faithful module with Krull dimension, then $\beta(A)$ is nilpotent. Let $a(M)=\{r \in R \mid r M=0\}$. Obviously $a(M)$ is an ideal of $R$ and $M$ is a faithful $R / a(M)$-module with Krull dimension. Hence by Markov's theorem, $\beta(R / a(M))$ is nilpotent. Clearly $(\beta(R)+a(M)) / a(M) \subseteq$ $\beta(R / a(M))$. This implies that there exists an $n$ such that $(\beta(R))^{n} \subseteq a(M)$, so $(\beta(R))^{n} M=0$. However $(\beta(R))^{n}$ is a $*$-ideal of $R$ and $M$ is a $*$-faithful $R$-module, so $(\beta(R))^{n}=0$.

We shall also need the following general results which were proved in [2].

Proposition 2. Let $S$ be a subring of a ring $R$ and let $I$ be an ideal of $R$ such that $I \subseteq S$. Suppose that every $(R / I)$-module has the same Krull dimension when considered as an $(R / I)$-module and as an $(S / I)$-module. Then every $R$-module has the same Krull dimension as an $R$-module and as an $S$-module.

Proposition 3. Let $S$ be a subring of a ring $A$ and $I$ a nilpotent ideal of $A$. Then $k_{(S+I)}(M)=k_{S}(M)$, for every $(S+I)$-module $M$.

\section{$\S 1$. Some Reductions}

In this section we make some auxiliary reductions.

Proposition 4. Suppose that there exists an $R$-module $M$ such that for some ordinal $\alpha, k_{R}(M)=\alpha$ but $k_{\bar{S}(R)}(M) \neq \alpha$. Then we can assume that $\bar{S}(R)=S(R)$ is a central subring of $R$ and $R$ is a semiprime PI-ring.

Proof. By [6] (Proof of Lemma 1.3), the ideal $I$ of $R$ generated by $\{x y-y x \mid x, y \in$ $\bar{S}(R)\}$ is contained in $\bar{S}(R)$. Clearly, $I$ is a $*$-ideal of $R$, so $S(R / I)=(S(R)+I) / I=$ $\bar{S}(R) / I$. Applying Proposition 2 we can pass to the factor ring $R / I$ and hence we can assume that $S(R)=\bar{S}(R)$ is commutative. From the quoted Amitsur's result it follows that $R$ is a PI ring. Factoring out the sum $T$ of all $*$-ideals of $R$ annihilating $M$, we can assume that $M$ is $*$-faithful. Now applying Proposition 1 we get that $\beta(R)$ is nilpotent. Hence Proposition 3 implies that $k_{(S(R)+\beta(R))}(M) \neq \alpha$. Now $S(R / \beta(R))=(S(R)+\beta(R)) / \beta(R)$, so applying Proposition 2 we can factor out $\beta(R)$ and assume that $R$ is semiprime. In [10], page 406, Lanski proved that if $S(R)$ is commutative, then for every $s \in S(R)$, the ideal of $R$ generated by $\{s r-r s \mid r \in R\}$ is nilpotent. This and semiprimeness of $R$ imply that $S(R)$ is central.

Proposition 5. Suppose that there exists an Artinian R-module which is not Artinian as an $\bar{S}(R)$-module. Then we can assume that $R$ is semiprime and $S(R)=$ $\bar{S}(R)$ is a central subring of $R$ such that every $0 \neq s \in S(R)$ is regular in $R$ and satisfies $s M=M$.

Proof. By Proposition 4 we can assume that $S(R)=\bar{S}(R)$ is a central subring of $R$. Obviously we can assume that all proper submodules of $M$ are Artinian as $S(R)$-modules. These properties do not change when passing to the factor ring 
$R / T$, where $T$ is the sum of all $*$-ideals of $R$ annihilating $M$. Thus we can assume that $M$ is a $*$-faithful $R$-module. By Proposition $1, \beta(R)$ is nilpotent.

Since $M$ is a $*$-faithful $R$-module and $S(R)$ is a central subring of $R$, for every $s \in S, a(s)=\{m \in M \mid s m=0\}$ is a proper $R$-submodule of $M$. Consequently $a(s)$ is an Artinian $S(R)$-module. The $R$-modules $M / a(s)$ and $s M$ are isomorphic, so $s M$ cannot be a proper submodule of $M$. Thus $s M=M$.

Now let $I=\{r \in R \mid r s=0\}$. Clearly, $I$ is a $*$-ideal of $R$. Moreover $I M=$ $I(s M)=(I s) M=0$. Since $M$ is a $*$-faithful $R$-module, $I=0$. This shows that $s$ is regular in $R$.

Since $\beta(R)$ is nilpotent, $\beta(R) M \neq M$. Hence $\beta(R) M$, being a proper $R$ submodule of $M$, is Artinian as an $S(R)$-module. Consequently $\bar{M}=M / \beta(R) M$ is an Artinian $R / \beta(R)$-module, which is not Artinian as an $(S(R)+\beta(R)) / \beta(R)=$ $S(R / \beta(R))$-module. Clearly, for every $0 \neq \bar{s}=s+\beta(R) \in S(R / \beta(R))$, we have $\bar{s} \bar{M}=\bar{M}$. Moreover $I=\{r \in R \mid r s \in \beta(R)\}$ is a $*$-ideal of $R$ such that $I^{n} s^{n}=(I s)^{n}=0$ for some $n$. Since $s$ is regular in $R$, we have $I^{n}=0$. This shows that $\bar{s}$ is regular in $R / \beta(R)$. Consequently we can replace $R$ by $R / \beta(R)$ obtaining a ring with the desired properties.

The following was proved in [2], Proposition 1.

Proposition 6. Let $S$ be a subring of a ring A. Suppose there exist an ordinal $\alpha \neq 0$ and an $A$-module $M$ such that $k_{A}(M)=\alpha$ but $k_{S}(M) \neq \alpha$, and suppose further that $\alpha$ is the least ordinal with this property. Then the module $M$ contains an $R$-submodule $K$ such that $k_{S}(K) \not \leq \alpha$ and, for every $R$-submodule $N$ of $K$, either $k_{S}(N) \leq \alpha$ or $k_{S}(K / N)<\alpha$.

Now we shall prove

Proposition 7. Suppose that $R$ has Krull dimension and that there exist an ordinal $\alpha$ and $R$-module $M$ with $k_{R}(M)=\alpha$ and $k_{\bar{S}(R)}(M) \neq \alpha$. We can assume that

(i) $R$ is a *-prime ring and $S(R)=\bar{S}(R)$ is a central subring of $R$;

(ii) for every $0 \neq c \in S(R), k_{S(R)}(M / c M)<\alpha$.

Proof. Suppose that we have chosen $R$ with the least possible Krull dimension $\gamma$ and then $M$ an $R$-module with the least possible Krull dimension $\alpha$. Observe that $\gamma>0$. Indeed, if $\gamma=0$, then $R$ is an Artinian ring. Hence $R$ is a Noetherian ring and by [2], for every $R$-module $M, k_{R}(M)=k_{\bar{S}(R)}(M)$.

Note that all the reductions in Proposition 4 do not increase the Krull dimension of the ring. Thus we can apply the proposition and assume that $S(R)=\bar{S}(R)$ is a central subring of $R$. Moreover, since $R$ has Krull dimension, $\beta(R)$ is nilpotent (here we could also apply arguments used in the proof of Proposition 5). Similarly as in the proof of Proposition 4, applying Propositions 3 and 2, we can factor out $\beta(R)$ and assume that $R$ is a semiprime ring. Since $R$ has Krull dimension, it has only a finite number of minimal prime ideals ([5], Proposition 7.3). Note that if $P$ is a prime ideal of $R$, then $P \cap P^{*}$ is a $*$-prime ideal of $R$. Consequently there are $*$-prime ideals $P_{1}, \ldots, P_{n}$ of $R$ such that $P_{1} \cap \ldots \cap P_{n}=0$. Let $M_{i}=P_{i} P_{i-1} \ldots P_{1} M, 1 \leq i \leq n$. Note that $0=M_{n} \subseteq M_{n-1} \subseteq \ldots \subseteq M_{1} \subseteq M_{0}=M$ and all $M_{i}$ are $R$-submodules of $M$. Hence for some $0 \leq i \leq n-1, k_{S(R)}\left(M_{i} / M_{i+1}\right) \not \leq \alpha$. Now $M_{i+1}=P_{i+1} M_{i}$, so $k_{S(R)}\left(M_{i} / M_{i+1}\right)=k_{\left(S(R)+P_{i+1}\right) / P_{i+1}}\left(M_{i} / M_{i+1}\right)=k_{S\left(R / P_{i+1}\right)}\left(M_{i} / M_{i+1}\right)$. Obviously $k_{R / P_{i+1}}\left(M_{i} / M_{i+1}\right) \leq \alpha$ and $S\left(R / P_{i+1}\right)$ is a central subring of $R / P_{i+1}$. This proves (i). 
Since $R$ is *-prime and $S(R)$ is central, each non-zero element $c \in S(R)$ is regular in $R$. Hence the Krull dimension of $R / c R$ is strictly smaller than $\gamma$. By the choice of $\gamma, k_{R / c R}(M / c M)=k_{S(R / c R)}(M / c M)=k_{(S(R)+c R) / c R}(M / c M)$. Obviously $k_{R / c R}(M / c M)=k_{R}(M / c M) \leq \alpha$ and, since $S(R / c R)=(S(R)+c R) / c R$, $k_{R / c R}(M / c M)=k_{S(R)}(M / c M)$. Thus $k_{S(R)}(M / c M) \leq \alpha$. This implies that $k_{S(R)}(c M) \not \leq \alpha$. Hence by Proposition 6, we may assume that $k_{S(R)}(M / c M)<\alpha$. The proof is complete.

\section{$\S 2$. Main Results}

We shall need the following results.

Proposition 8 ([14, Theorem 7.6]). Let $\sigma$ be an automorphism of order 2 of a ring $A$ with $1 / 2 \in A$ and let $A^{\sigma}=\{a \in A \mid \sigma(a)=a\}$ be the fixed subring of $A$ with respect to $\sigma$. Then $k_{A}(M)=k_{A^{\sigma}}(M)$ for every $A$-module $M$.

Proposition 9 ([7, Theorem 3.10]). Suppose that $R$ is non-commutative and semiprime and $S=S(R)$ is a field. Then

(i) $S$ is the center of $R$;

(ii) $R$ is a division ring with $[R: S]=4$ or $R$ is isomorphic to the ring $M_{2}(S)$ of $2 \times 2$ - matrices over $S$.

Proposition 10 ([13, Theorem 1]. Suppose that $K$ is a field of characteristic $\neq 2$ and $*$ is a $K$-involution of the ring $M_{2}(K)$. Then, in an appropriate $K$-basis, $* i s$ one of the following types:

$$
\begin{aligned}
& \text { (transpose involution) }\left(\begin{array}{ll}
a & b \\
c & d
\end{array}\right)^{*}=\left(\begin{array}{ll}
a & b \\
c & d
\end{array}\right) \\
& \text { or } \\
& \text { (symplectic involution) }\left(\begin{array}{ll}
a & b \\
c & d
\end{array}\right)=\left(\begin{array}{cc}
d & -b \\
-c & a
\end{array}\right)
\end{aligned}
$$

for all $a, b, c, d$ in $K$.

In the former case, $S\left(M_{2}(K)\right)$ is not a subring of $M_{2}(K)$.

Now we can prove

Theorem 1. Suppose that $M$ is an R-module. Suppose further that

(i) $R$ is semiprime, $S=S(R)$ is a central subring of $R$ and every non-zero element of $S$ is regular in $R$;

(ii) $k_{R}(M)=\alpha$ and for every $0 \neq s \in S, k_{S}(M / s M)<\alpha$.

Then $k_{S}(M)=\alpha$.

Proof. Suppose the theorem is not true and $\alpha$ is the least possible ordinal for which it does not hold. Obviously $\alpha>-1$. By Proposition 6 we can assume that $M$ is an $R$-module such that $k_{R}(M)=\alpha, k_{S}(M) \neq \alpha$ and for every $R$-submodule $N$ of $M, k_{S}(N) \leq \alpha$ or $k_{S}(M / N)<\alpha$.

We claim that for every $0 \neq c \in S, k_{S+c R}(M) \leq \alpha$. If not, then there is a strictly descending chain $\left\{M_{i} \mid i=0,1,2, \ldots\right\}$ of $(S+c R)$-submodules of $M$ such that $M_{0}=M$ and for every $i \geq 1, k_{(S+c R)}\left(M_{i-1} / M_{i}\right) \nless \alpha$. This in particular implies that for $N=R M_{1}, k_{S}(N) \not \leq \alpha$. Since $N$ is an $R$-submodule of $M$, the assumption on $M$ we made at the beginning of the proof gives that $k_{S}(M / N)<\alpha$. Consequently $k_{S}(c M / c N)<\alpha$. By (ii), $k_{S}(M / c M)<\alpha$, so $k_{S}(M / c N)<\alpha$. However $c N=c R M_{1} \subseteq M_{1}$, so $k_{S}\left(M_{0} / M_{1}\right)=k_{S}\left(M / M_{1}\right) \leq k_{S}(M / c M)<\alpha$. This contradiction proves the claim. 
If $R$ is commutative, then $*$ is an automorphism of $R$ of order 2 the fixed ring of which is equal $S$. We get a contradiction with Proposition 8. Thus $R$ must be non-commutative. We can form the ring $Q=S^{-1} R$ of quotients of $R$ with respect to the non-zero elements of $S$. The involution can be extended uniquely to $Q$ and then $K=S(Q)$ is the field of fractions of the domain $S$. Now Propositions 9 and 10 allow us to assume that $Q$ is the ring of $2 \times 2$ - matrices over $K$ with the symplectic involution or that $Q$ is a division ring with center $K$ and $[Q: K]=4$.

We claim that there exists a skew-symmetric element $x \in R$ such that $x^{2} \neq 0$ and $B=\{z \in Q \mid z x=x z\}$ is equal to the $K$-subalgebra $K[x]$ of $Q$ generated by $x$. Suppose first that $Q$ is a division ring. Since 2 is invertible in $R$, for every $0 \neq r \in R \backslash S, x=r-r^{*}$ is a skew-symmetric element of $R$ not belonging to $K$. Obviously $x^{2} \neq 0$. Since $x^{2} \in K$ and $x \notin K$, $\operatorname{dim}_{K}(K[x])=2$. Therefore $K[x]$ is a maximal subfield of $Q$ and $K[x]=B$. Suppose now that $Q$ is the ring of $2 \times 2$ - matrices over $K$ with the symplectic involution. Then $Q$ contains a set $E=\left\{e_{i j} \mid i, j=1,2\right\}$ of matrix units such that $e_{12}^{*}=-e_{12}$ and $e_{21}^{*}=-e_{21}$. Clearly $y=e_{12}+e_{21}$ is a skew-symmetric invertible element of $Q$. There exists $0 \neq s \in S$ such that $x=s y \in R$. Obviously $x$ is a skew-symmetric element of $R$ and $x^{2} \neq 0$. Direct computations show that $B=K[x]$. The claim is proved.

Let $\sigma$ be the inner automorphism of $Q$ induced by $x$. Since $x^{2} \in S$ is a central element of $R$ (and so of $Q$ ), $\sigma^{2}=i d_{Q}$. We also note that $\sigma$ commutes with the involution. Observe that for $s=x^{2}, A=S+s R+x R x$ is a $\sigma$-invariant and $*$-invariant subring of $R$. We already know that $k_{S+s R}(M) \leq \alpha$. Since the lattice of $A$-submodules of $M$ is a sublattice of the lattice of $(S+s R)$-submodules of $M$, we conclude that $k_{A}(M) \leq \alpha$. By Proposition 8 we have that $k_{A}(M)=$ $k_{A^{\sigma}}(M)$. Clearly $A^{\sigma} \subseteq B=\{z \in Q \mid z x=x z\}$ and so $A^{\sigma}$ is a commutative ring. Recalling that $\sigma$ commutes with $*$, we conclude that $A^{\sigma}$ is a $*$-invariant subring of $R$ containing $S$. Therefore $*$ induces an automorphism of order two on $A^{\sigma}$. Noting that $S\left(A^{\sigma}\right)=S$ and again applying Proposition 8, we infer that

$$
k_{S}(M)=k_{A^{\sigma}}(M)=k_{A}(M) \leq \alpha .
$$

Since the lattice of $R$-submodules of $M$ is a sublattice of the lattice of $S$-submodules of $M$, we conclude that $k_{R}(M) \leq k_{S}(M)$. Thus $k_{S}(M)=\alpha$ which contradicts our assumption. The proof is complete.

Theorem 1 and Proposition 5 give

Corollary 1. Every Artinian R-module is Artinian as an $\bar{S}(R)$-module.

Applying Theorem 1 and Proposition 7 we get

Corollary 2. If $R$ is a ring with Krull dimension and $M$ is an $R$-module, then $k_{R}(M)=k_{\bar{S}(R)}(M)$.

We close with a result which sheds some further light on Lanski's general problem. Note that in particular it shows that the general question can be reduced to the study of $*$-faithful modules over semiprime Goldie rings.

Theorem 2. Suppose that $R$ contains an invertible skew-symmetric element $x$. Then for every $R$-module $M, k_{R}(M)=k_{\bar{S}(R)}(M)$.

Proof. Suppose that there exists an $R$-module $M$ with $k_{R}(M) \neq k_{\bar{S}(R)}(M)$. Applying Proposition 4 we can assume that $R$ is a semiprime PI ring and $S=\bar{S}(R)$ is a central subring of $R$. Factoring out the sum of all $*$-ideals of $R$ annihilating 
$M$, we can assume that $M$ is $*$-faithful. Now by Proposition 1 we get that $\beta(R)$ is nilpotent. Set $I=\beta(R), I^{0}=R, M_{t}=I^{t} M / I^{t+1} M$ and $N=\bigoplus_{t=0}^{n} M_{t}$, where $I^{n+1}=0$. We claim that $N$ is a $*$-faithful $R / I$-module. Indeed, if $J$ is a $*$-ideal of $R$ such that $(J / I) N=0$, then $J I^{t} M \subseteq I^{t+1} M$ for $t=0,1, \ldots, n$. It follows that $J^{n+1} M \subseteq I^{n+1} M=0$. Hence, since $M$ is $*$-faithful, $J^{n+1}=0$. Therefore $J=I$, which proves the claim. Now passing to the ring $R / I$ and the module $N$ and applying Propositions 2 and 3 we can assume that $R$ is semiprime and $M$ is *-faithful. Let $A=\{r \in R \mid r M=0\}$ be the annihilator of $M$. Clearly $A$ is an ideal of $R$ and $M$ is a faithful $R / A$-module. Hence by [12], the prime radical $B / A$ of $R / A$ is nilpotent, i.e., $B^{m+1} \subseteq A$ for a non-negative integer $m$. Similarly as above one checks that $N=\bigoplus_{t=0}^{m} B^{t} M / B^{t+1} M$ is a faithful $R / B$-module. It is known [3] that every semiprime PI ring having a faithful module with Krull dimension is a (left and right) Goldie ring. Hence $R / B$ is a Goldie ring. Note that since $M$ is a *-faithful $R$-module, $A \cap A^{*}=0$. Moreover $\left(B \cap B^{*}\right)^{m+1} \subseteq A \cap A^{*}=0$. Hence since $R$ is semiprime, $B \cap B^{*}=0$. Now $R / B^{*}$ is antiisomorphic to $R / B$, so $R / B^{*}$ is a Goldie ring. Since $B$ is isomorphic to the ideal $\left(B+B^{*}\right) / B^{*}$ of $R / B^{*}, B$ is also a Goldie ring. Let $I=\{r \in R \mid r B=0\}$ be the annihilator of $B$ in $R$. Since $R$ is semiprime, $I \cap B=0$. Hence $I$ is isomorphic to the ideal $(I+B) / B$ of $R / B$, so $I$ is a Goldie ring. Finally $I+B=I \oplus B$ is a Goldie ring which is an essential ideal of $R$. Hence $R$ is a Goldie ring. Let $T$ be the set of all regular elements in $S$. Note that if $c$ is a regular central element of $R$, then $c^{*}$ and $c c^{*}$ are regular central elements of $R$ and $c c^{*} \in T$. Hence regular central elements of $R$ are invertible in the classical ring of quotients $Q=T^{-1} R$. Now by Proposition 5.8 in [15], $Q$ is a semisimple Artinian ring, so $Q=\bigoplus_{i=1}^{n} A_{i}$, where $A_{i}$ are simple rings. We can extend the involution from $R$ to $Q$ putting $(r / t)^{*}=r^{*} / t$, for $r \in R, t \in T$. Then $S(Q)=T^{-1} S$ and hence $S(Q)$ is a central subring of $Q$. Obviously for every $1 \leq i \leq n$ there is $1 \leq j \leq n$ such that $A_{i}^{*}=A_{j}$. If $i \neq j$, then commutativity of $S(Q)$ implies that both $A_{i}$ and $A_{j}$ are fields. If $i=j$, then by Propositions 9 and $10, A_{i}$ is isomorphic to the ring of $2 \times 2$-matrices over a field with the symplectic involution or it is a division ring of dimension 4 over its center. In both cases $S\left(A_{i}\right)$ coincides with the center of $A_{i}$.

Denote by $\sigma$ the inner automorphism of $R$ induced by $x$. Since $x^{2} \in S$ is a central element, $\sigma^{2}=i d_{R}$. We claim that $R^{\sigma}$ is a commutative subring of $R$. Indeed, it is enough to show that $Q^{\sigma}$ is a commutative subring of $Q$. The foregoing show that it suffices to prove the claim in the case when $Q$ is the ring of $2 \times 2$-matrices over a field with the symplectic involution or $Q$ is a division ring of dimension 4 over its center. We can reduce the latter case to the former by tensoring $Q$ by the algebraic closure of its center. Thus we can assume that $Q=M_{2}(F)$, where $F$ is an algebraically closed field and $S(Q)=F$. Since $x^{2} \in F$, we conclude that the Jordan normal form of $x$ is $\left(\begin{array}{cc}c & 0 \\ 0 & -c\end{array}\right)$ for some $0 \neq c \in F$. It follows that $Q^{\sigma}$ is isomorphic to

a subring of the ring $\left(\begin{array}{ll}F & 0 \\ 0 & F\end{array}\right)$, which is obviously commutative. The claim is proved. Note now that $R^{\sigma}$ is a $*$-invariant subring of $R$ and $S=\left\{r \in R^{\sigma} \mid r^{*}=r\right\}$. Clearly * induces on $R^{\sigma}$ an automorphism of order two. Applying twice Proposition 8 we get that $k_{R}(M)=k_{S}(M)$, a contradiction. The proof is complete.

\section{REFERENCES}

1. S.A. Amitsur, Rings with involution, Israel J. Math. 6 (1968), 99-106. MR 39:256

2. K.I. Beidar, E.R. Puczyłowski and P.F. Smith, Krull dimension of modules over involution rings, Proc. Amer. Math. Soc. 121 (1994), 391-397. MR 94h:16064 
3. K.I. Beidar and V.T. Markov, A semiprime PI-ring having a faithful module with Krull dimension is a Goldie ring, Russian Math. Survey 48 (1993), 141-142. MR 94m:16023

4. C.L. Chuang and P.H. Lee, Noetherian rings with involution, Chinese J. Math. 5 (1977), 15-19. MR 56:12053

5. R. Gordon and J.C. Robson, Krull dimension, Memoirs Amer. Math. Soc., No. 133, American Mathematical Society, Providence, 1973. MR 50:4664

6. I.N. Herstein, Topics in ring theory, Univ. Chicago Press, Chicago, 1969. MR 42:6018

7. C. Lanski, On the relationship of a ring and the subring generated by its symmetric elements, Pacific J. Math. 44 (1973), 581-592. MR 48:331

8. C. Lanski, Chain conditions in rings with involution, J. London Math. Soc. 9 (1974), 93-102. MR 50:13123

9. C. Lanski, Chain conditions in rings with involution II, J. London Math. Soc. 18 (1978), 421-428. MR 80a:16024

10. C. Lanski, Gabriel dimension and rings with involution, Houston Math. J. 4 (1978), 397-415. MR 80a:16025

11. P.H. Lee On subrings of rings with involution, Pacific J. Math. 60 (1975), 131-147. MR 53:519

12. V.T. Markov, On PI rings having a faithful module with Krull dimension (to appear).

13. S. Montgomery, A structure theorem and a positive-definiteness condition in rings with involution, J. Algebra 43 (1976), 181-192. MR 54:12821

14. S. Montgomery, Fixed rings of finite automorphism groups of associative rings, Lectures Notes in Math. Vol. 818, Springer-Verlag, Berlin-Heidelberg-New York, 1980. MR 81j:16041

15. L.H. Rowen, On rings with central polynomials, J. Algebra 31 (1974), 393-426. MR 50:2237

Department of Mathematics, Moscow State University, Moscow, Russia

Current address: National Cheng-Kung University, Department of Mathematics, Tainan, Taiwan

E-mail address: t14270@sparc1.cc.ncku.edu.tw

Institute of Mathematics, University of Warsaw, Warsaw, Poland

E-mail address: edmundp@mimuw.edu.pl

Department of Mathematics, University of Glasgow, Glasgow, Scotland

E-mail address: pfs@maths.gla.ac.uk 